\title{
Recognition of the organ-specific mutations in metastatic breast cancer by circulating tumor cells isolated in vivo
}

\author{
Sainan $\mathrm{LI}^{1}$, Shan YANG ${ }^{1}$, Jiajie SHI ${ }^{1}$, Yawen DING ${ }^{1}$, Wei GAO ${ }^{1}$, Meng CHENG ${ }^{1}$, Yuqiao SUN ${ }^{1}$, Yanli XIE ${ }^{1}$, Meixiang SANG ${ }^{2}$, Hongxia YANG ${ }^{3}$, Cuizhi GENG ${ }^{1, *}$ \\ ${ }^{1}$ Breast Center Department, The Fourth Hospital of Hebei Medical University, Shijiazhuang, Hebei, China; ${ }^{2}$ Research Center and Tumor Re- \\ search Institute, The Fourth Hospital of Hebei Medical University, Shijiazhuang, Hebei, China; ${ }^{3}$ Beijing Viroad Biotechnology Co., Ltd. Beijing, \\ China
}

*Correspondence: cuizhigeng@hotmail.com

Received March 17, 2020 / Accepted July 10, 2020

\begin{abstract}
The failure to treat and control the growth of metastases is the main cause of death in breast cancer (BC) patients. Compared to the traditional method of analyzing circulating tumor DNA (ctDNA), capturing intact circulating tumor cells (CTCs) allows us to more accurately characterize mutations and identify suitable targeted therapies. We used CellCollector to collect peripheral CTCs. Thirty metastatic breast cancer (MBC) patients were enrolled, and 17 were analyzed with nextgeneration sequencing (NGS) methods. Clinical characteristics were analyzed along with the CTCs enumeration and detection rates. Whole-genome amplification (WGA) was used to amplify the CTC genomic DNA of 127 genes. Patients younger than 45 years old, with brain metastasis, with three or more metastatic sites, or with HER2-positive had the highest number of CTCs collected. The CTCs detection rate was also correlated to the number of metastasis sites. Different metastasis sites such as the brain, viscus, bone, and soft tissue contained specific high-frequency gene mutations. AKT3, MYC, and NT5C2 mutations were only found in brain metastases. APC, BCL2L11, ESRP1, FLT3 mutations were only in the visceral metastases. KEAP1, KIT, MET were the specific mutation genes in patients with bone and soft tissue metastases. These findings provide evidence that we can detect gene mutation information for obtaining the biological characteristics by CTCs using CellCollector. Different metastasis sites contain specific high-frequency mutation genes, which provide guidance to the accurate gene therapy.
\end{abstract}

Key words: CellCollector, in vivo, circulating tumor cells, metastatic breast cancer, gene mutations

Despite recent advances in diagnostic and treatment strategies, breast cancer (BC) still accounts for 271,270 deaths per year worldwide [1]. Failures to treat and control metastases are the main causes of mortality from BC. The development of metastasis in patients is believed to result from tumor cells entry into the circulation and the subsequent migration to distant sites [2, 3], where the mutation landscape of metastatic breast cancer (MBC) may be different from those of the primary tumor. Therefore, acquiring biological information of metastatic loci is essential for individualized therapies. Tissue biopsies, the most commonly used diagnostic method, obtain only limited information due to sampling bias from spatial heterogeneities of tumors $[4,5]$. Moreover, some metastatic foci are technically challenging or dangerous to access, such as brain metastases, limiting the biological information available. Patients also have to bear risks and more complications from biopsy procedures.
Therefore, researchers and physicians have turned to circulating tumor DNA (ctDNA) and circulating tumor cells (CTCs) for assessing the metastatic tumor as the procedures are convenient, well-tolerated, and minimally invasive [6]. ctDNA is a small fraction of DNA originating from cancerous cells and tumors [7], whose mutation and abundance provide information on tumor mutation and efficiency of treatment respectively [8]. However, only limited information could be obtained due to the fragmented nature of ctDNA. On the other hand, CTCs are intact tumor cells circulating in the blood, and if successfully isolated, provide much richer information on tumor cells in terms of protein markers, wholegenome analysis, and comprehensive characterization of mutations [9]. BC is heterogeneous, whether it is between the primary and metastatic foci, or between different metastatic sites or different cells that constitute the same metastatic foci. CTC-based gene mutation detection and analysis can 
monitor metastatic burden in real time to assist clinical treatment decisions $[10,11]$.

Currently, CellSearch is the typical method to capture CTCs, and had lots of clinical research in BC, prostate cancer, and colorectal cancer [12-15]. However, due to the limited volume of blood analyzed, CellSearch fails to capture and separate intact CTCs, which restricts its clinical applications and limits downstream sequencing to identify mutations in metastatic tissues. To overcome this limitation, we used CellCollector, an in vivo CTC-capturing device [16], to collect whole CTCs from MBC patients. Isolated CTCs were then subjected to whole-genome amplification and sequencing to obtain genetic information [17]. To our best knowledge, studies about BC on CTC-specific gene mutations obtained via CellCollector have not yet been published.

In this study, we captured and isolated intact CTCs from MBC patients. Subsequent whole-genome sequencing analysis of these CTCs revealed mutations specific to different metastatic organs, including the brain. These results could provide guidance to individualized therapies.

\section{Patients and methods}

Study design. This was a single-center prospective study. A total of 30 patients with verified MBC and 30 healthy volunteers were enrolled in the study. Patients who were 18 years or older, diagnosed as MBC by biopsy or imaging, had an Eastern Cooperative Oncology Group (ECOG) score for performance status from 0 to 2 , and no second malignancies were enrolled. All samples were collected at the Fourth Hospital of Hebei Medical University, China. Before the study, written informed consent was obtained from each patient.

Collection of CTCs through CellCollector. The CellCollector (GILUPI GmbH, Potsdam, Germany) device is a stainless-steel wire with antibodies against the epithelial cell surface antigen EpCAM attached to a polycarboxylate/ hydrogel coated on a gold-plated layer. CellCollector captures target cells expressing the EpCAM antigen on the membrane of different cancer cell types (Figure 1).

The device was inserted into the median cubital vein to a manufacturer indicated length through a $20 \mathrm{G}$ peripheral venous catheter until the functional domain was exposed

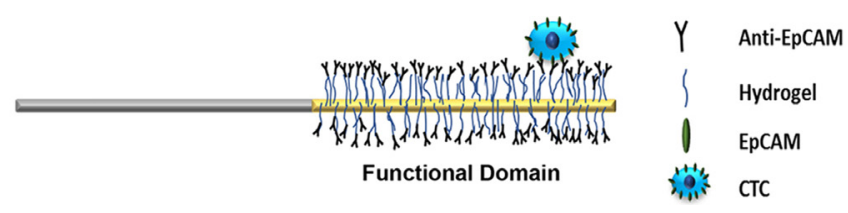

Figure 1. Mechanism of CTC detection. The CellCollector device is a stainless steel wire with antibodies against the epithelial cell surface antigen EpCAM attached to a polycarboxylate/hydrogel coated on a goldplated layer and captures target cells expressing the EpCAM antigen on the membrane of different cancer cell types. to blood flow and securely fixed to the intravenous cannula. The wire remained in the cubital vein for $30 \mathrm{~min}$ application period and was estimated to collect CTCs from $1500 \mathrm{ml}$ of blood.

Confirmation of CTCs through immunofluorescence. After application, the device was gently washed in wash buffer and blocked in PBS with $2 \%(\mathrm{w} / \mathrm{v})$ bovine serum albumin (BSA) for $30 \mathrm{~min}$ at room temperature. CTCs captured by CellCollector were identified by immunofluorescence staining for EpCAM or cytokeratins 8, 18, and 19. Cells attached to the wire were incubated with a FITCconjugated mouse monoclonal antibody directed against EpCAM (Acris, clone HEA125-FITC) and an APC-conjugated rabbit antibody raised against CD45 (Exbio, clone MEM-28-Alexa647). Cells were counterstained with the nuclear dye Hoechst 33342 (Sigma, catalog number H6024). Immunofluorescence was detected using an Axio Imager, Alm microscope (Zeiss, Jena, Germany) equipped with an AxioCam digital camera system and AxioVision 4.6 software (Zeiss). EpCAM/cytokeratin-positive cells typically exhibited additional features, including a large cell body (diameter 10-50 $\mu \mathrm{m})$, an irregular cell shape, a large irregularly shaped nucleus, and a high nuclear to cytoplasmic ratio. CD45 staining was used to identify white blood cells. EpCAM/ CK-positive, nuclear positive, and CD45-negative cells were identified as CTCs, and EpCAM/CK-negative, nuclear positive, and CD45-positive cells were identified as leukocytes. Patients with one or more CTCs were determined to be CTC positive.

Genome extraction and amplification. Under the fluorescence microscope, the CellCollector fragments with CTCs were clipped and transferred into a $0.2 \mathrm{ml}$ PCR tube. And a single CellCollector fragment is $<1 \mathrm{~mm}$. The human genome DNA was diluted to $30 \mathrm{pg} / \mathrm{ml} .1 \mu \mathrm{l}$ was transferred into a PCR tube containing $3 \mu \mathrm{lPBS}$ as a positive control. $4 \mu \mathrm{l}$ PBS was used as a non-template control and a small section of CellCollector without cells was cut to a PCR tube containing $4 \mu \mathrm{PBS}$ as a negative control. Whole-genome amplification (WGA) for CTC samples and control was conducted simultaneously. Cell lysis and WGA were performed using REPLI-g Single Cell Kit (Qiagen, catalog number 150343) following the manufacturer's instructions.

After diluting the amplified product 20 times, $1 \mu$ was used to measure the dsDNA concentration by Qubit 3.0. Generally, the total amount of product was about $35 \mu \mathrm{g} / 30 \mu \mathrm{l}$ of the reaction system. Negative control and no template control had DNA below 50 ng. The qualified CTC genome amplification products were subjected to downstream gene mutation analysis.

Sequencing library construction and data analysis. Approximately $300 \mathrm{ng}$ of amplified products were used for library construction, quality inspection, and next-generation sequencing (NGS). NGS was performed with HiSeq X Ten (Illumina) following manufacturer protocols using pairedend 150 bp (PE150) sequencing strategy with a 127-gene 
panel. Paired-end sequences were mapped to reference human genome (hg19) using BWA. Candidate somatic SNV and indel less than $50 \mathrm{bp}$ were called using Pisces. After annotation to databases including oncoKB, Oncomine, and Cosmic 80, candidate translocation breakpoints were analyzed and filtered using seeksv and BreakTrans.

\section{Results}

Patient characteristics. Thirty eligible patients were enrolled from December 2016 to May 2017. Patients' demographics and disease characteristics are detailed in Table 1. There were 14 patients who had one metastasis site including 9 local recurrences, 2 bone metastasis, 2 liver metastasis, and 1 lung metastasis. Sixteen patients had two or more different metastasis sites. Patients were divided into three groups based on the metastatic sites (brain metastasis, visceral metastasis, and bone/soft tissue metastasis). For patients with more than one metastatic site, grouping was based on the invasiveness of the metastasis organs. For example, patients with brain and lung metastasis would be categorized as brain metastasis group.

CTCs and correlations with clinical characteristics. All enrolled 30 patients were performed in vivo CTCs detection before systemic therapy, and 30 healthy individuals served as the control group. Examples of EpCAM-positive CTCs captured by the device are shown in Figure 2. In total, 66.7\% (20/30) MBC patients had more than one CTC, and numbers of CTCs collected ranged from 0 to 15 (median $=2$, mean $=2$ ). No CTC was observed in the control group (Figure 3). All patients tolerated the device insertion without any adverse events.

The numbers of CTCs seemed correlated with clinical characteristics (Table 2 and Figure 4). Patients who were $<45$ years old, HER2 positive, brain metastasis, with three metastasis sites or more had the highest median and mean CTCs number in their corresponding subgroups. Of the 3 brain metastasis patients, who had the highest CTCs numbers, we detected a mean of 6 CTCs and a median of 5 CTCs (range, $0-15$ ). We did not capture CTCs in the luminal A group. No statistical analysis was carried out due to the small number of patients.

More metastasis sites seemed to lead to higher CTCs detection rates, and the group of three metastasis sites or more had $100 \%$ detection. $<45$ years, HER2 positive groups had the highest CTCs rates in their corresponding subgroups (Figure 4).

Subtype and metastatic site-specific mutations identified in CTCs. Genomic DNA was successfully amplified from CTCs from 17 patients. NGS of 127 genes to analyze single nucleic acid variation (SNV) and insertions/deletions (IN/DELs). If more than half of the patients had the same gene mutation, the gene is considered a high-frequency mutation gene (HFGM). In total, 19 HFGMs were identified from the luminal B group, 52 in the HER2 positive group,
Table 1. Demographic and clinicopathological features of the $30 \mathrm{MBC}$ patients.

\begin{tabular}{lcc}
\hline Patient characteristics & Case (n) & Percentage (\%) \\
\hline Age (years) & & \\
$\quad<45$ & 12 & 40.0 \\
$\geq 45$ and $<60$ & 13 & 43.3 \\
$\quad \geq 60$ & 5 & 16.7 \\
Metastatic location & & \\
$\quad$ Brain & 3 & 10.0 \\
$\quad$ Lung + liver & 14 & 46.7 \\
$\quad$ Bone + local recurrence & 13 & 43.3 \\
Number of metastatic locations & & \\
1 & 14 & 46.7 \\
2 & 6 & 20.0 \\
3-4 & 10 & 33.3 \\
Molecular subtypes & & \\
Luminal A & 1 & 3.3 \\
Luminal B & 11 & 36.7 \\
HER2 positive & 11 & 36.7 \\
Triple-negative & 7 & 23.3 \\
\hline
\end{tabular}

Abbreviations: MBC-metastatic breast cancer; HER2-human epidermal growth factor receptor 2

Table 2. Number of CTCs collected and correlations with clinicopathological characteristics.

\begin{tabular}{|c|c|c|c|}
\hline \multirow{2}{*}{ Patients' characteristics } & \multicolumn{3}{|c|}{ CTCs enumeration } \\
\hline & Range & median & mean \\
\hline Total & $0-15$ & 2 & 2 \\
\hline \multicolumn{4}{|l|}{ Age (years) } \\
\hline$<45$ & $0-4$ & 2 & 2 \\
\hline$\geq 45$ and $<60$ & $0-15$ & 1 & 2 \\
\hline$\geq 60$ & $0-2$ & 1 & 2 \\
\hline \multicolumn{4}{|l|}{ Metastatic location } \\
\hline Brain & $0-15$ & 5 & 6 \\
\hline Lung + liver & $0-7$ & 1 & 2 \\
\hline Bone +local recurrence & $0-4$ & 1 & 1 \\
\hline \multicolumn{4}{|c|}{ Number of metastatic locations } \\
\hline 1 & $0-4$ & 0 & 1 \\
\hline 2 & $0-15$ & 1 & 3 \\
\hline $3-4$ & $1-7$ & 2 & 3 \\
\hline \multicolumn{4}{|l|}{ Molecular subtypes } \\
\hline Luminal A & 0 & 0 & 0 \\
\hline Luminal B & $0-5$ & 1 & 1 \\
\hline HER2 positive & $0-15$ & 2 & 3 \\
\hline Triple-negative & $0-7$ & 1 & 2 \\
\hline
\end{tabular}

Abbreviations: CTCs-Circulating Tumor Cells; HER2-human epidermal growth factor receptor 2

and 82 in the triple-negative group. Some gene mutations occurred only in specific subgroups (Figure 5A). We did not analyze HFGMs in the luminal A group as no CTCs were captured from patients in this group.

An important finding in this study is that different metastasis sites have their own corresponding HFGMs. 41 specific gene mutations occurred in brain metastasis patients, in 

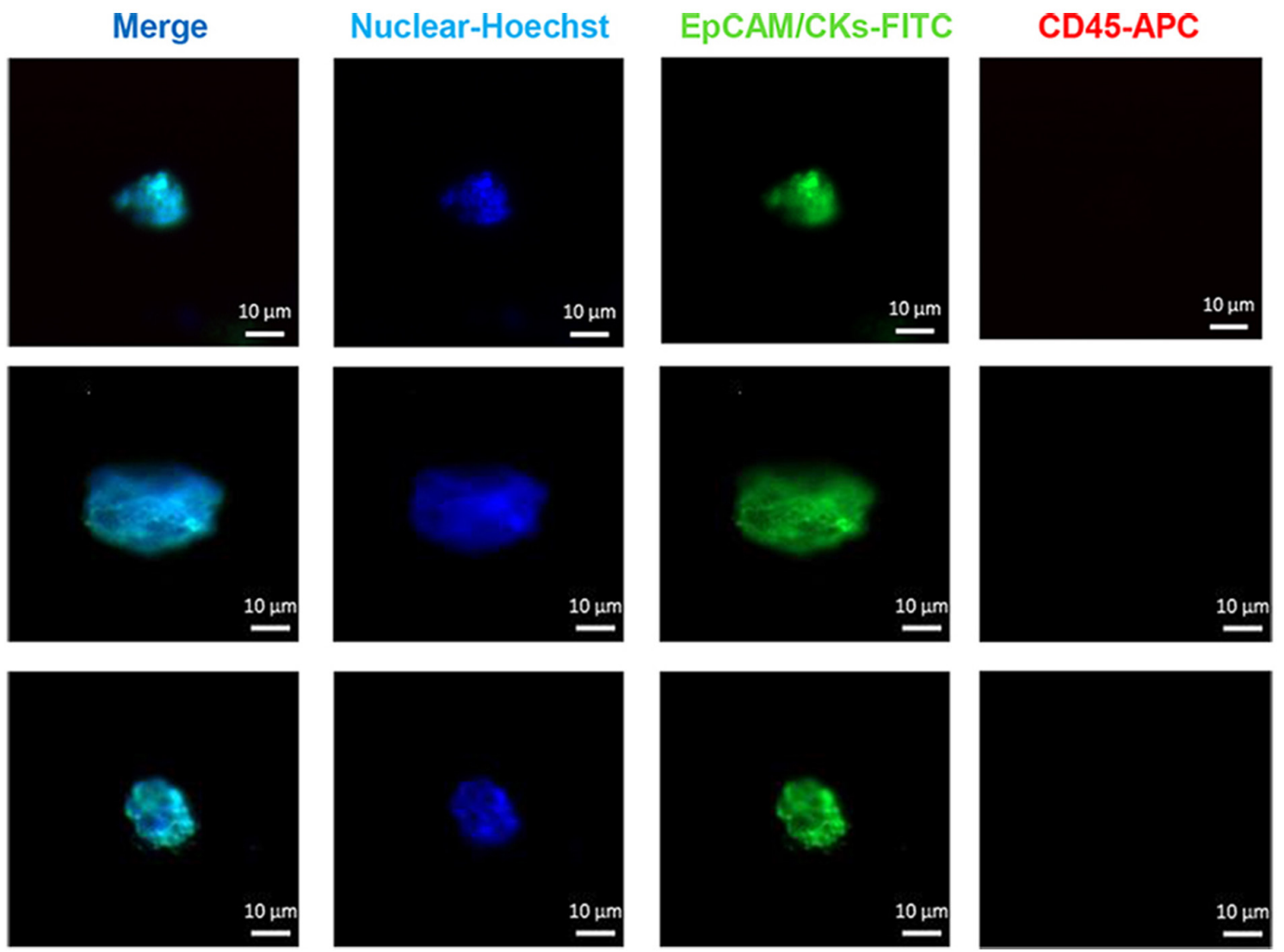

Figure 2. Immunofluorescence analysis of CTCs in vivo with CellCollector. Nucleated cells with EpCAM/CKs expression but no CD45 expression, and intact cell morphology are considered CTC positive and counted. The CTCs examples shown represent 3 different MBC patients.

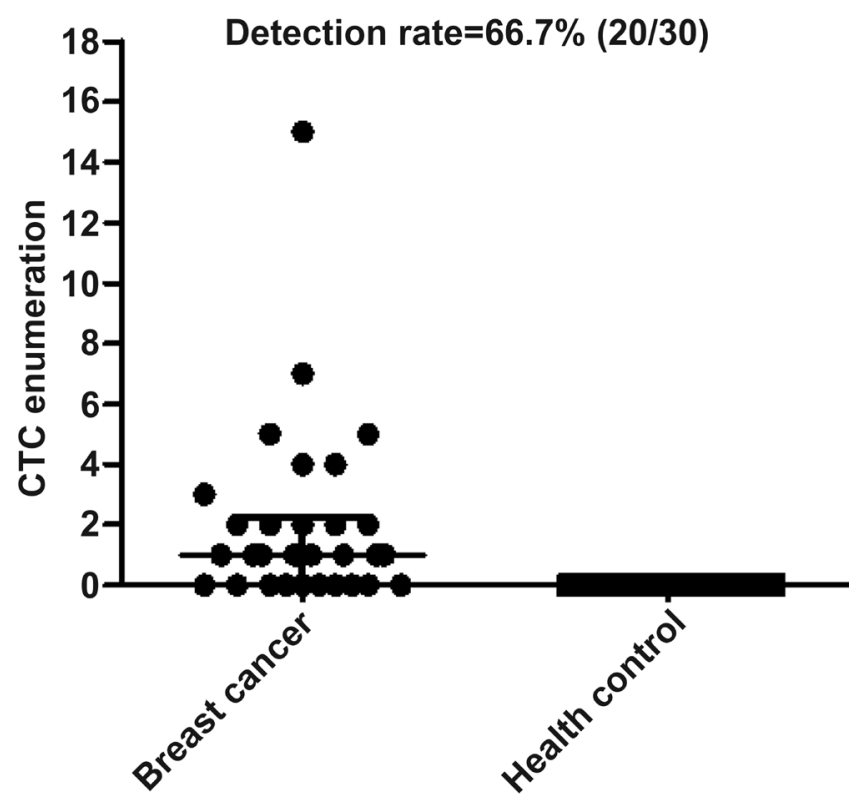

Figure 3. CTCs enumeration in $\mathrm{BC}$ and healthy control patients. Scatterplot of all CellCollector applications. $66.7 \%(20 / 30)$ patients were detected $>1$ CTC, no CTC was detected in the 30 control patients. which 19 gene mutations such as ATM, MTOR, MYC, RET, etc. had never been reported before. 71 specific gene mutations were found in visceral metastasis patients (Figure 5B), and 67 specific gene mutations were detected in bone metastasis and local recurrence patients (Figure 5B). Notably, each group had its own specific HFGMs. For example, mutated genes such as $A K T 3, M Y C$, and NT5C2 appeared only in the brain metastasis group (Figure 5C). APC, BCL2L11, ESRP1, FLT3, PIK3CA, STAT3, and TPM3 were only in the viscera metastasis group, and KEAP1, KIT, and MET were mutant genes in bone metastasis and local recurrence patients.

\section{Discussion}

The spread of tumor cells to peripheral blood is an important stage in distant metastasis, which is the main cause of death in many cancer patients. Sensitive and specific detection and characterization of CTCs is essential for the understanding of these metastatic cells. Here, we employed a new in vivo CTC detection device, CellCollector, to detect tumor cells in the peripheral blood of MBC patients. We detected CTCs in $66.7 \%$ of MBC patients recruited, higher than the representative detection rate ranging from $57.4 \%$ to $65 \%$ by 

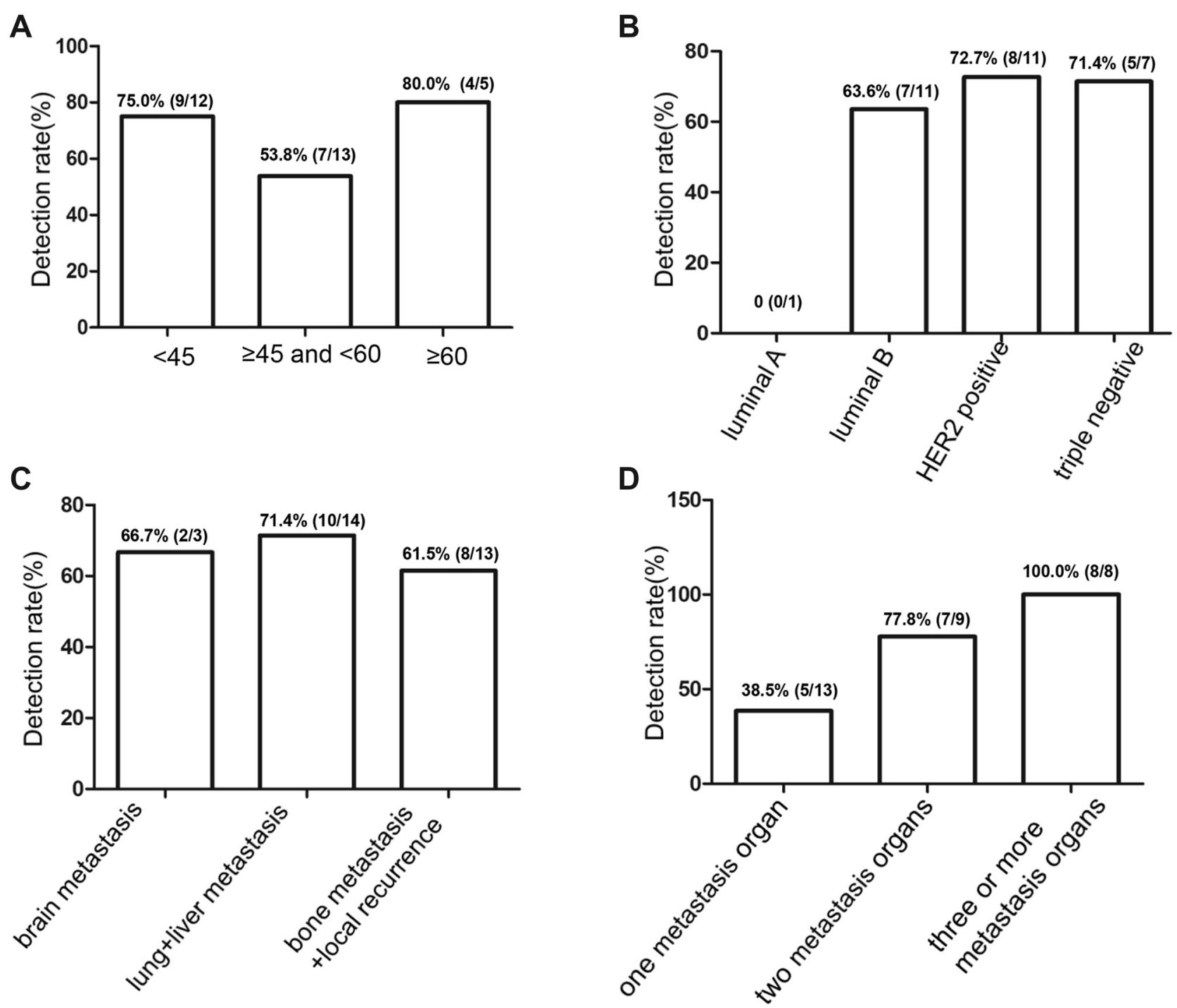

Figure 4. CTCs detection rates in different groups. A) CTCs detection rates in different age groups. B) CTCs detection rates in different molecular subtype groups. C) CTCs detection rates in different metastatic location groups. D) CTCs detection rates in a different number of metastasis site groups.

CellSearch [18]. The superiority of CellCollector was also shown in research done in lung cancer and prostate cancer patients [17, 19, 20]. Moreover, CTCs captured using this in vivo assay can be easily isolated for downstream molecular characterization including NGS, which could provide additional genetic information useful for future targeted therapy developments [17, 21, 22].

The numbers of CTCs collected and detection rates were correlated with clinical characteristics. Patients with brain metastasis had the most CTCs detected. Additionally, there were more CTCs in patients younger than 45 years, with HER2- positive subtype, and with multiple metastatic sites. In the one, two, and three or more metastasis organs groups, the CTCs detection rates were $38.5 \%, 77.8 \%$, and $100.0 \%$, respectively. This close association with the number of metastases is consistent with findings in non-small-cell lung cancer and gestational choriocarcinoma $[6,23]$. We propose that worse prognostic factors, such as younger age, aggressive molecular subtype, and widespread metastasis may affect the CTCs numbers. Unfortunately, due to the limited number of subjects enrolled, our study was not sufficiently powered for further statistical analysis.

At present, genomic information from bulk primary or metastatic tumors could guide individualized therapies and de-escalated treatment for a better quality of life and improved survival outcomes. However, multiple studies have shown that tumors have spatiotemporal heterogeneity, and genomic analysis from a single tumor biopsy specimen may underestimate the tumor genome diversity, limiting the efficacy of personalized treatments and the discovery of 

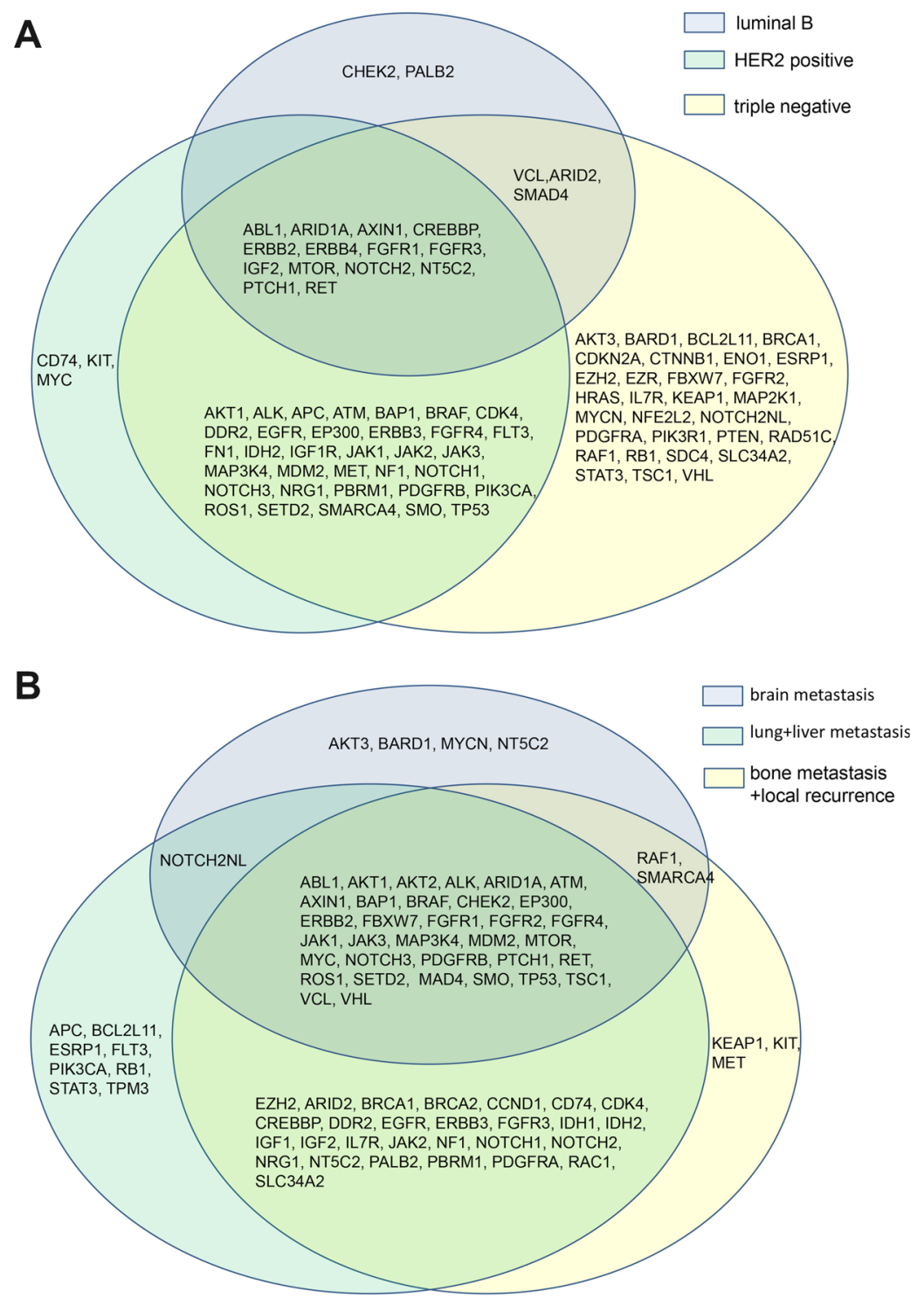

C

\begin{tabular}{|c|c|c|c|}
\hline AKT3 & JAK1 & PTCH1 & VHL \\
\hline ARID1A & JAK3 & SETD2 & \\
\hline AXIN1 & MAP3K4 & SMAD4 & \\
\hline BAP1 & MYC & SMARCA4 & \\
\hline BARD1 & NOTCH3 & TSC1 & \\
\hline CHEK2 & NT5C2 & VCL & \\
\hline
\end{tabular}

Figure 5. High-frequency gene mutations in different molecular subtypes and metastasis organ groups. A) High-frequency gene mutations in different molecular subtype groups. Blue, luminal B breast cancer group. Green, HER2 positive breast cancer group. Yellow, triple-negative breast cancer group. B) High-frequency gene mutations in different metastasis sites groups. Blue, brain metastasis group. Green, lung + liver metastasis group. Yellow, bone metastasis + local recurrence group. C) High-frequency gene mutations, which had never been reported in brain metastasis before. biomarkers [24-26]. Reiter et al. conducted a comprehensive evaluation of untreated tumor metastases and found a large set of driver mutations common to all metastases [27]. This may be due to the small number of initial tumor cells planted at each metastatic site, or limited biopsy sampling. Future clinical studies of cancer metastasis should consider liquid biopsy methods, such as CTC capturing. The importance of molecular information revealed by CTCs is further emphasized by a recent study, where CTCs have higher mutation overlap with metastatic tissue compared to the primary tumor [28].

In this study, we characterized the mutational landscape of CTCs and analyzed the association between mutations and clinical tumor manifestations. To the best of our knowledge, such an investigation in MBC patients to date is rare. Notably, in our study, there are highfrequency mutant genes in MBC patients common to many metastatic organs. One such example is FGFR, whose aberrations can lead to tumor, proliferation, and metastasis. Previously, it has been reported that $18 \%$ of BC patients had FGFR abnormalities [29]. With the clinical development of FGFR pathway inhibitors underway, this information could guide targeted therapies in the future. Moreover, we found HFGMs specific to MBC patients with different metastatic organs and molecular types including triple-negative breast cancer (TNBC). TNBC is highly invasive. Due to the lack of effective drug targets, the main treatment strategy at present is chemotherapy, with the worst outcomes among all BC subtypes. We found a BRCA1 mutation in the CTC of a TNBC patient, which may indicate that this patient could be sensitive to platinum drugs or PARP inhibitors [30, 31]. Of course, this requires a large sample of prospective clinical studies to confirm. Moreover, Jiao et al. result showed that the PPARgamma agonist pioglitazone had an inhibition effect on the proliferation and migration of BC cells, which was correlated with the JAK2/STAT3 pathway [32].

$\mathrm{BC}$ patients have a high risk of brain metastases, second only to lung cancer. Recent studies have shown that BC cells have genetic, molecular, and metabolic changes and have the ability to survive in 
the brain microenvironment $[33,34]$. We found that TP53 mutation exists in patients with brain metastasis breast cancer (BCBM), which is consistent with previous studies. TP53 mutations are frequently observed in up to $60 \%$ of BCBM and have a higher frequency of mutations in TNBC and HER2+BC subgroups $[35,36]$. We also found HFGMs specifically present in BCBM in our cohort, including $A K T 3$, $B A R D 1, M Y C N, N T 5 C 2$. Grottke et al. showed that downregulation of $A K T 3$ expression can promote the invasion and metastasis ability of triple-negative $\mathrm{BC}$ [37], and brain metastases had significantly higher P-AKT expression than distant metastases from the lung and liver [38]. The implicated that, the DNA repair gene BARD1 is frequently overexpressed in brain metastases from $\mathrm{BC}$ and may constitute a mechanism to overcome reactive oxygen species-mediated genotoxic stress in the metastatic brain [39]. MYCN is upregulated in $\mathrm{BCBM}$ and could be a molecular biomarker identification in BCBM [40].

During metastasis, some tumor cells retain their biological characteristics, while others manifest mutations different from those in the primary tumor [26]. Moreover, CTCs might harbor specific mutations for migrating to and surviving in different destinations with different microenvironments. In this study, we found mutated genes in MBC patients' CTCs showing clusters according to the organ with metastasis found. Understanding CTC mutations could allow early detection of metastatic cancer [41, 42]. More investigations and analysis of data from larger populations are required to reveal any metastatic organ-specific mutations in CTCs and their link to mechanisms of metastasis.

In conclusion, CellCollector has a high CTC detection rate and can perform diverse downstream gene mutation detection. CTC may be associated with the number of metastatic sites and metastatic organs, HER2 status, and age. Different metastasis sites may contain specific HFMGs and some of which have never been reported before. Moreover, AKT3, $M Y C$, and NT5C2 mutations were only found in brain metastasis. APC, BCL2L11, ESRP1, FLT3 mutations were only in visceral metastasis. And KEAP1, KIT, MET were the specific mutation genes in bone and soft tissue metastasis patients in this study.

This is a new attempt to detect gene mutation information by CTC. Whether the mutated genes affect tumor metastasis and the role of these mutations in the tumor invasion process remains to be further explored in subsequent studies, and then ultimately to guide therapy.

Acknowledgments: This work was supported by a grant from the marathon fund of Beijing.

\section{References}

[1] SIEGEL RL, MILLER KD, JEMAL A. Cancer statistics, 2019. CA Cancer J Clin 2019; 69: 7-34. https://doi.org/10.3322/ caac. 21551
[2] SCHMITT MW, LOEB LA, SALK JJ. The influence of subclonal resistance mutations on targeted cancer therapy. Nat Rev Clin Oncol 2016; 13: 335-347. https://doi.org/10.1038/ nrclinonc.2015.175

[3] YU M, STOTT S, TONER M, MAHESWARAN S, HABER DA. Circulating tumor cells: approaches to isolation and characterization. J Cell Biol 2011; 192: 373-382. https://doi. org/10.1083/jcb.201010021

[4] SAUNDERS NA, SIMPSON F, THOMPSON EW, HILL MM, ENDO-MUNOZ L et al. Role of intratumoural heterogeneity in cancer drug resistance: molecular and clinical perspectives. EMBO Mol Med 2012; 4: 675-684. https://doi. org/10.1002/emmm.201101131

[5] PANTEL K, ALIX-PANABIERES C. Circulating tumour cells in cancer patients: challenges and perspectives. Trends Mol Med 2010; 16: 398-406. https://doi.org/10.1016/j.molmed.2010.07.001

[6] KREBS MG, SLOANE R, PRIEST L, LANCASHIRE L, HOU JM et al. Evaluation and prognostic significance of circulating tumor cells in patients with non-small-cell lung cancer. J Clin Oncol 2011; 29: 1556-1563. https://doi.org/10.1200/ JCO.2010.28.7045

[7] STROUN M, ANKER P, MAURICE P, LYAUTEY J, LEDERREY C et al. Neoplastic characteristics of the DNA found in the plasma of cancer patients. Oncology 1989; 46: 318-322. https://doi.org/10.1159/000226740

[8] PAPADOPOULOS N. Pathophysiology of ctDNA Release into the Circulation and Its Characteristics: What Is Important for Clinical Applications. Recent Results Cancer Res 2020; 215: 163-180. https://doi.org/10.1007/978-3-03026439-0_9

[9] TODENHOFER T, PANTEL K, STENZL A, WERNER S. Pathophysiology of Tumor Cell Release into the Circulation and Characterization of CTC. Recent Results Cancer Res 2020; 215: 3-24. https://doi.org/10.1007/978-3-030-26439-0_1 [10] SHAW JA, GUTTERY DS, HILLS A, FERNANDEZ-GARCIA D, PAGE K et al. Mutation Analysis of Cell-Free DNA and Single Circulating Tumor Cells in Metastatic Breast Cancer Patients with High Circulating Tumor Cell Counts. Clin Cancer Res 2017; 23: 88-96. https://doi.org/10.1158/10780432.CCR-16-0825

[11] BYSTRICKY B, MEGO M. Circulating tumor cells in breast cancer patients. Neoplasma 2016; 63: 18-29. https://doi. org/10.4149/neo_2016_003

[12] LIANIDOU E, PANTEL K. Liquid biopsies. Genes Chromosomes Cancer 2019; 58: 219-232. https://doi.org/10.1002/ gcc. 22695

[13] COHEN SJ, PUNT CJ, IANNOTTI N, SAIDMAN BH, SABBATH KD et al. Prognostic significance of circulating tumor cells in patients with metastatic colorectal cancer. Ann Oncol 2009; 20: 1223-1229. https://doi.org/10.1093/annonc/ $\operatorname{mdn} 786$

[14] COHEN SJ, PUNT CJ, IANNOTTI N, SAIDMAN BH, SABBATH KD et al. Relationship of circulating tumor cells to tumor response, progression-free survival, and overall survival in patients with metastatic colorectal cancer. J Clin Oncol 2008; 26: 3213-3221. https://doi.org/10.1200/ JCO.2007.15.8923 
[15] VAN DALUM G, VAN DER STAM GJ, TIBBE AG, FRANKEN B, MASTBOOM WJ et al. Circulating tumor cells before and during follow-up after breast cancer surgery. Int J Oncol 2015; 46: 407-413. https://doi.org/10.3892/ijo.2014.2694

[16] SAUCEDO-ZENI N, MEWES S, NIESTROJ R, GASIOROWSKI L, MURAWA D et al. A novel method for the in vivo isolation of circulating tumor cells from peripheral blood of cancer patients using a functionalized and structured medical wire. Int J Oncol 2012; 41: 1241-1250. https:// doi.org/10.3892/ijo.2012.1557

[17] GORGES TM, PENKALLA N, SCHALK T, JOOSSE SA, RIETHDORF $S$ et al. Enumeration and Molecular Characterization of Tumor Cells in Lung Cancer Patients Using a Novel In Vivo Device for Capturing Circulating Tumor Cells. Clin Cancer Res 2016; 22: 2197-2206. https://doi. org/10.1158/1078-0432.CCR-15-1416

[18] MAHESWARAN S, HABER DA. Circulating tumor cells: a window into cancer biology and metastasis. Curr Opin Genet Dev 2; 20: 96-99. https://doi.org/10.1016/j.gde.2009.12.002

[19] CHEN S, TAUBER G, LANGSENLEHNER T, SCHMOLZER LM, POTSCHER $M$ et al. In Vivo Detection of Circulating Tumor Cells in High-Risk Non-Metastatic Prostate Cancer Patients Undergoing Radiotherapy. Cancers (Basel) 2019; 11: 933. https://doi.org/10.3390/cancers11070933

[20] KUSKE A, GORGES TM, TENNSTEDT P, TIEBEL AK, POMPE $\mathrm{R}$ et al. Improved detection of circulating tumor cells in non-metastatic high-risk prostate cancer patients. Sci Rep 2016; 6: 39736. https://doi.org/10.1038/srep39736

[21] EL-HELIEBI A, HILLE C, LAXMAN N, SVEDLUND J, HAUDUM C et al. In Situ Detection and Quantification of AR-V7, AR-FL, PSA, and KRAS Point Mutations in Circulating Tumor Cells. Clin Chem 2018; 64: 536-546. https://doi. org/10.1373/clinchem.2017.281295

[22] DUAN GC, ZHANG XP, WANG HE, WANG ZK, ZHANG H et al. Circulating Tumor Cells as a Screening and Diagnostic Marker for Early-Stage Non-Small Cell Lung Cancer. Onco Targets Ther 2020; 13: 1931-1939. https://doi.org/10.2147/ OTT.S241956

[23] HE W, HOU M, ZHANG H, ZENG C, HE S et al. Clinical significance of circulating tumor cells in predicting disease progression and chemotherapy resistance in patients with gestational choriocarcinoma. Int J Cancer 2019; 144: 14211431. https://doi.org/10.1002/ijc.31742

[24] MCGRANAHAN N, SWANTON C. Clonal Heterogeneity and Tumor Evolution: Past, Present, and the Future. Cell 2017; 168: 613-628. https://doi.org/10.1016/j.cell.2017.01.018

[25] PRIESTLEY P, BABER J, LOLKEMA MP, STEEGHS N, DE BRUIJN E et al. Pan-cancer whole-genome analyses of metastatic solid tumours. Nature 2019; 575: 210-216. https://doi. org/10.1038/s41586-019-1689-y

[26] NG CKY, BIDARD FC, PISCUOGLIO S, GEYER FC, LIM RS et al. Genetic Heterogeneity in Therapy-Naive Synchronous Primary Breast Cancers and Their Metastases. Clin Cancer Res 2017; 23: 4402-4415. https://doi.org/10.1158/1078-0432. CCR-16-3115

[27] REITER JG, MAKOHON-MOORE AP, GEROLD JM, HEYDE A, ATTIYEH MA et al. Minimal functional driver gene heterogeneity among untreated metastases. Science 2018; 361: 1033-1037. https://doi.org/10.1126/science.aat7171
[28] CHEMI F, ROTHWELL DG, MCGRANAHAN N, GULATI S, ABBOSH C et al. Pulmonary venous circulating tumor cell dissemination before tumor resection and disease relapse. Nat Med 2019; 25: 1534-1539. https://doi.org/10.1038/ s41591-019-0593-1

[29] HELSTEN T, ELKIN S, ARTHUR E, TOMSON BN, CARTER J et al. The FGFR Landscape in Cancer: Analysis of 4,853 Tumors by Next-Generation Sequencing. Clin Cancer Res 2016; 22: 259-267. https://doi.org/10.1158/1078-0432.CCR14-3212

[30] GEENEN JJJ, LINN SC, BEIJNEN JH, SCHELLENS JHM. PARP Inhibitors in the Treatment of Triple-Negative Breast Cancer. Clin Pharmacokinet 2018; 57: 427-437. https://doi. org/10.1007/s40262-017-0587-4

[31] PAPADIMITRIOU M, MOUNTZIOS G, PAPADIMITRIOU CA. The role of PARP inhibition in triple-negative breast cancer: Unraveling the wide spectrum of synthetic lethality. Cancer Treat Rev 2018; 67: 34-44. https://doi.org/10.1016/j. ctrv.2018.04.010

[32] JIAO XX, LIN SY, LIAN SX, QIU YR, LI ZH et al. The inhibition of the breast cancer by PPARgamma agonist pioglitazone through JAK2/STAT3 pathway. Neoplasma 2020; 67: 834-842. https://doi.org/10.4149/neo_2020_190805N716

[33] MEDRESS Z, HAYDEN GEPHART M. Molecular and Genetic Predictors of Breast-to-Brain Metastasis: Review and Case Presentation. Cureus 2015; 7: e246. https://doi. org/10.7759/cureus.246

[34] BROSNAN EM, ANDERS CK. Understanding patterns of brain metastasis in breast cancer and designing rational therapeutic strategies. Ann Transl Med 2018; 6: 163. https:// doi.org/10.21037/atm.2018.04.35

[35] LEE JY, PARK K, LIM SH, KIM HS, YOO KH et al. Mutational profiling of brain metastasis from breast cancer: matched pair analysis of targeted sequencing between brain metastasis and primary breast cancer. Oncotarget 2015; 6: 43731-43742. https://doi.org/10.18632/oncotarget.6192

[36] CANCER GENOME ATLAS N. Comprehensive molecular portraits of human breast tumours. Nature 2012; 490: 61-70. https://doi.org/10.1038/nature11412

[37] GROTTKE A, EWALD F, LANGE T, NORZ D, HERZBERGER C et al. Downregulation of AKT3 Increases Migration and Metastasis in Triple Negative Breast Cancer Cells by Upregulating S100A4. PLoS One 2016; 11: e0146370. https:// doi.org/10.1371/journal.pone.0146370

[38] DAVIES MA, STEMKE-HALE K, LIN E, TELLEZ C, DENG $\mathrm{W}$ et al. Integrated Molecular and Clinical Analysis of AKT Activation in Metastatic Melanoma. Clin Cancer Res 2009; 15: 7538-7546. https://doi.org/10.1158/1078-0432.CCR-091985

[39] WODITSCHKA S, EVANS L, DUCHNOWSKA R, REED LT, PALMIERI D et al. DNA double-strand break repair genes and oxidative damage in brain metastasis of breast cancer. J Natl Cancer Inst 2014; 106: dju145. https://doi.org/10.1093/ jnci/dju 145

[40] SCHULTEN HJ, BANGASH M, KARIM S, DALLOL A, HUSSEIN D et al. Comprehensive molecular biomarker identification in breast cancer brain metastases. J Transl Med 2017; 15: 269. https://doi.org/10.1186/s12967-017-1370-x 
[41] NI X, ZHUO M, SU Z, DUAN J, GAO Y et al. Reproducible copy number variation patterns among single circulating tumor cells of lung cancer patients. Proc Natl Acad Sci U S A 2013; 110: 21083-21088. https://doi.org/10.1073/ pnas. 1320659110
[42] EBRIGHT RY, LEE S, WITTNER BS, NIEDERHOFFER KL, NICHOLSON BT et al. Deregulation of ribosomal protein expression and translation promotes breast cancer metastasis. Science 2020;367(6485):1468-1473. https://doi. org/10.1126/science.aay0939 